\title{
Sex difference in top performers from Ironman to double deca iron ultra-triathlon
}

This article was published in the following Dove Press journal:

Open Access Journal of Sports Medicine

26 June 2014

Number of times this article has been viewed

\author{
Beat Knechtle' \\ Matthias A Zingg 2 \\ Thomas Rosemann ${ }^{2}$ \\ Christoph A Rüst ${ }^{2}$ \\ 'Gesundheitszentrum St Gallen, \\ St Gallen, Switzerland; ${ }^{2}$ Institute \\ of General Practice and Health \\ Services Research, University of \\ Zurich, Zurich, Switzerland
}

Correspondence: Beat Knechtle Gesundheitszentrum St Gallen, Vadianstrasse 26, St Gallen 900I, Switzerland

$\mathrm{Tel}+4|7| 2268282$

Fax $+4 \mid$ 7| 2268272

Email beat.knechtle@hispeed.ch
Abstract: This study investigated changes in performance and sex difference in top performers for ultra-triathlon races held between 1978 and 2013 from Ironman $(3.8 \mathrm{~km}$ swim, $180 \mathrm{~km}$ cycle, and $42 \mathrm{~km}$ run) to double deca iron ultra-triathlon distance ( $76 \mathrm{~km}$ swim, 3,600 km cycle, and $844 \mathrm{~km}$ run). The fastest men ever were faster than the fastest women ever for split and overall race times, with the exception of the swimming split in the quintuple iron ultra-triathlon (19 km swim, $900 \mathrm{~km}$ cycle, and $210.1 \mathrm{~km}$ run). Correlation analyses showed an increase in sex difference with increasing length of race distance for swimming $\left(r^{2}=0.67, P=0.023\right)$, running $\left(r^{2}=0.77, P=0.009\right)$, and overall race time $\left(r^{2}=0.77, P=0.0087\right)$, but not for cycling $\left(r^{2}=0.26\right.$, $P=0.23$ ). For the annual top performers, split and overall race times decreased across years nonlinearly in female and male Ironman triathletes. For longer distances, cycling split times decreased linearly in male triple iron ultra-triathletes, and running split times decreased linearly in male double iron ultra-triathletes but increased linearly in female triple and quintuple iron ultra-triathletes. Overall race times increased nonlinearly in female triple and male quintuple iron ultra-triathletes. The sex difference decreased nonlinearly in swimming, running, and overall race time in Ironman triathletes but increased linearly in cycling and running and nonlinearly in overall race time in triple iron ultra-triathletes. These findings suggest that women reduced the sex difference nonlinearly in shorter ultra-triathlon distances (ie, Ironman), but for longer distances than the Ironman, the sex difference increased or remained unchanged across years. It seems very unlikely that female top performers will ever outrun male top performers in ultratriathlons. The nonlinear change in speed and sex difference in Ironman triathlon suggests that female and male Ironman triathletes have reached their limits in performance.

Keywords: triathlon, swimming, cycling, running, ultra-endurance

\section{Introduction}

Triathlon is a multisport discipline consisting of the three disciplines swimming, cycling, and running, held in this order. ${ }^{1}$ The distances range from the sprint distance triathlon (750 m swim, $20 \mathrm{~km}$ cycle, and $5 \mathrm{~km}$ run) to the double deca iron ultra-triathlon distance (78 km swim, 3,600 km cycle, and $844 \mathrm{~km}$ run. ${ }^{2,3}$ Long-distance triathlon is an interesting model to investigate the sex difference in ultra-endurance performance because the sex difference in performance can be examined for each split discipline such as swimming, cycling, and running, and also for overall race time. ${ }^{4}$ Since the first Ironman distance triathlon held in Hawaii in $1978,{ }^{5}$ the ultra-distance triathlon has been expanded to the double deca iron ultra-triathlon distance, and the first race was held in 1998 in Monterrey, Mexico. ${ }^{3}$

In recent years, the sex difference in elite triathlon performance has been investigated for sprint distance triathlon (750 m swim, $20 \mathrm{~km}$ cycle, and $5 \mathrm{~km}$ run), ${ }^{2}$ 
short-distance triathlon $(1.5 \mathrm{~km}$ swim, $40 \mathrm{~km}$ cycle, and $10 \mathrm{~km}$ run $),{ }^{6}$ Ironman triathlon $(3.8 \mathrm{~km}$ swim, $180 \mathrm{~km}$ cycle, and $42.195 \mathrm{~km}$ run $){ }^{7}$ double iron ultra-triathlon $(7.6 \mathrm{~km}$ swim, $360 \mathrm{~km}$ cycle, and $84.4 \mathrm{~km}$ run), ${ }^{8}$ and triple iron ultra-triathlon (11.4 km swim, $540 \mathrm{~km}$ cycle, and $126.6 \mathrm{~km}$ run). ${ }^{9}$ The sex differences in performance seemed to be different for the different race distances. For short-distance triathletes competing in the International Triathlon Union World Triathlon Series during the 2009-2012 period, including the 2012 London Olympic games, the sex difference in performance remained unchanged for swimming and cycling but decreased for running and for overall race time across years. ${ }^{6}$ The sex difference in running was greater compared to swimming and cycling. ${ }^{6}$ In the annual ten fastest women and men competing in Ironman Hawaii, between 1983 and 2012, the sex difference decreased across years in overall race time and running but not in swimming and cycling. ${ }^{7}$ In double iron ultra-triathletes competing between 1985 and 2012, the sex difference in performance remained unchanged in the three split disciplines and in overall race time. ${ }^{8}$ In triple iron ultra-triathletes competing between 1988 and 2011, the sex difference increased in the three split disciplines and in overall race time. ${ }^{9}$

These studies investigated mainly the annual three or the annual ten fastest women and men with the exception of triple iron ultra-triathletes, where only the annual winners were analyzed due to the low female participation in this distance. ${ }^{6-9}$ Additionally, these studies used linear regression analyses to investigate the trend in performance and sex difference over time. ${ }^{6-9}$ However, a linear change in both performance and sex difference in performance would mean that performances of men and women would intercept in the next decades and centuries, ie, women would outperform men at some point. However, sport as a whole shows a nonlinear improvement over time. ${ }^{10}$ Furthermore, the investigation of a fixed sample per year (ie, the annual ten fastest) fails to show the trend for the absolute top performers in the field. Additionally, analyses of the longer ultra-triathlon distances such as the quadruple iron ultra-triathlon (15.2 km swim, $720 \mathrm{~km}$ cycle, and $168.8 \mathrm{~km}$ run), the quintuple iron ultra-triathlon (19 km swim, $900 \mathrm{~km}$ cycle, and $210.1 \mathrm{~km}$ run), the deca iron ultratriathlon ( $38 \mathrm{~km}$ swim, 1,800 km cycle, and $422 \mathrm{~km}$ run), and the double deca iron ultra-triathlon (76 km swim, 3,600 km cycle, and $844 \mathrm{~km}$ run) are missing.

Recent studies investigating ultra-endurance athletes competing in single sport disciplines such as ultra-running, ${ }^{11,12}$ ultra-cycling, ${ }^{13,14}$ and ultra-swimming, ${ }^{15,16}$ using linear and nonlinear analyses, showed that the gap is narrowing between men and women in ultra-running ${ }^{11,12}$ and ultra-cycling, ${ }^{14}$ and women were faster than men in ultra-swimming. ${ }^{15}$ In ultraswimming, the gap was reduced between men and women in the $36 \mathrm{~km}$ Maratona del Golfo Capri-Napoli race held from 1954 to $2013^{17}$ and in La Traversée Internationale du Lac St Jean held between 1955 and 2012. ${ }^{17,18}$ In ultra-cycling, the gap between the sexes was reduced in the $720 \mathrm{~km}$ Swiss Cycling Marathon, the largest European qualifier for the Race Across America. ${ }^{14}$ In the Race Across America itself, the sex difference remained, however, unchanged at $\sim 19 \%$ for the annual fastest in the last 30 years. ${ }^{13}$ In ultra-running, the gap between women and men was reduced in 24-hour ultra-marathons. ${ }^{11}$ These findings for swimming, cycling, and running might allow the assumption that women would be able to achieve performance levels equal to men or even be able to outperform men in the longer ultra-triathlon distances (ie, longer than the triple iron ultra-triathlon).

Therefore, the present study investigated the changes in performance and sex difference in performance for the annual top performers for all ultra-triathlon distances from Ironman to double deca iron ultra-triathlons held between 1978 and 2013 using linear and nonlinear analyses. With this approach, we may be able to distinguish whether these athletes have reached their limits or not, and whether women might be able to close the gap with men in the near future in very long ultra-triathlon distances (longer than the triple iron ultra-triathlon) or even to outperform men.

\section{Materials and methods Ethics}

All procedures used in the study were approved by the Institutional Review Board of Kanton St Gallen, Switzerland with a waiver of the requirement for informed consent of the participants given the fact that the study involved the analysis of publicly available data.

\section{Data sampling and analysis}

Overall race times and split times in swimming, cycling, and running were collected from the Ironman World Championship Ironman Hawaii between 1978 and 2013 and from all ultra-triathlons (ie, longer than the Ironman distance) held between 1985 and 2013. Split and overall race times for Ironman Hawaii were obtained from the Ironman race website (http://www.ironmanworldchampionship.com). ${ }^{19}$ For the triathlon distances between double iron and double deca iron ultra-triathlon, data were obtained from the website of the International Ultra-Triathlon Association (IUTA) (http://www.iutasport.com) ${ }^{20}$ and from the race directors for 
earlier years not recorded in the IUTA website. The double iron ultra-triathlon held in Huntsville, AL, USA in 1994 had to be excluded from data analysis since that race included a downstream swimming split leading to unrealistically fast swimming split times.

Split times in swimming, cycling, and running and overall race times of the annual fastest finishers between 1978 and 2013 from Ironman Hawaii and all ultra-triathlons up to double deca iron ultra-triathlons held between 1985 and 2013 were analyzed. In the first step, we analyzed the performance and the sex difference in performance for split and overall race times of the fastest finishers ever for each distance. In the second step, we investigated the changes in performance and sex difference in performance across years of the annual top performers. To find sex differences in performance, split and overall race times of the annual top women and men were sorted by race distance. The sex difference was calculated using the equation,

$\frac{\text { Race time for women }- \text { Race time for men }}{\text { Race time for men }} \times 100$

where all race times are expressed in minutes. All sex differences were transformed to absolute values prior to statistical analysis.

\section{Statistical analysis}

Each set of data was tested for normal distribution and for homogeneity of variances prior to statistical analyses. Normal distribution was tested using a D'Agostino and Pearson omnibus normality test and homogeneity of variances was tested using a Levene's test. A potential relationship between the sex difference of the fastest competitors and both split and overall race times in men was investigated using Pearson's correlation analysis. To compare performance between two groups (eg, men versus women), a Student's $t$-test was used. To investigate changes in performance and sex difference in performance of the annual fastest, single and multilevel regression analyses were used. A hierarchical regression model was used to avoid the impact of a cluster-effect in case one athlete finished more than once in the annual top. Since the change in sex difference in endurance performance is assumed to be nonlinear, ${ }^{10}$ we calculated the linear and the nonlinear regression model for performance and sex difference in performance in swimming, cycling, running, and overall race time. In order to find the best model to explain the trend of the data, we compared the best-fit nonlinear model to the linear model using Akaike's information criteria and $F$-test. Statistical analyses were performed using SPSS Statistics (version 22; IBM Corporation, Armonk, NY, USA), CurveExpert Professional (version 2.0.3; Hyams DG) and GraphPad Prism (version 6.01; GraphPad Software, La Jolla, CA, USA). Significance was accepted at $P<0.05$ (two-sided for Student's $t$-tests). Data in the text and figures are given as mean \pm standard deviation.

\section{Results}

\section{Participation trends}

A total of 42,961 athletes (9,502 women and 33,459 men) finished Ironman Hawaii and ultra-triathlons longer than the Ironman Hawaii between 1978 and 2013 (Table 1). Women accounted on average for $11.9 \% \pm 5.8 \%$ of all athletes (Table 1). The percentage of female finishers was highest in Ironman Hawaii and lowest in deca iron ultra-triathlon (Table 1).

\section{Performance and sex difference in performance of the fastest finishers ever}

Figure 1 presents the split (Figure $1 \mathrm{~A}-\mathrm{C}$ ) and overall race times (Figure 1D) for the fastest competitors ever from Ironman to double deca iron ultra-triathlon. The fastest men ever were faster than the fastest women ever in both split and overall race times, with the exception of the swimming split time in the quintuple iron ultra-triathlon (Figure 1A), where the fastest female finisher ever was faster than the fastest male competitor ever. Figure 2 shows the sex

Table I Number of female and male finishers in all races

\begin{tabular}{|c|c|c|c|c|c|}
\hline Races and distances & $\begin{array}{l}\text { Female } \\
\text { finishers }\end{array}$ & $\begin{array}{l}\text { Male } \\
\text { finishers }\end{array}$ & $\begin{array}{l}\text { Overall } \\
\text { finishers }\end{array}$ & $\begin{array}{l}\text { Female } \\
\text { finishers (\%) }\end{array}$ & $\begin{array}{l}\text { Male } \\
\text { finishers (\%) }\end{array}$ \\
\hline Ironman Hawaii (1978-2013) & 9,502 & 33,459 & 42,961 & 22.1 & 77.9 \\
\hline Double iron ultra-triathlon (1985-2013) & 179 & 1,731 & 1,910 & 10.3 & 89.7 \\
\hline Triple iron ultra-triathlon (1988-2013) & 98 & 918 & 1,016 & 10.7 & 89.3 \\
\hline Quadruple iron ultra-triathlon (1989-2013) & 3 & 39 & 42 & 7.1 & 92.9 \\
\hline Quintuple iron ultra-triathlon (1991-2013) & 13 & 59 & 72 & 18.0 & 82.0 \\
\hline Deca iron ultra-triathlon (1992-2013) & 8 & 123 & 128 & 6.5 & 93.5 \\
\hline Double deca iron ultra-triathlon (I998-2013) & I & 10 & II & 9.1 & 90.1 \\
\hline
\end{tabular}


A

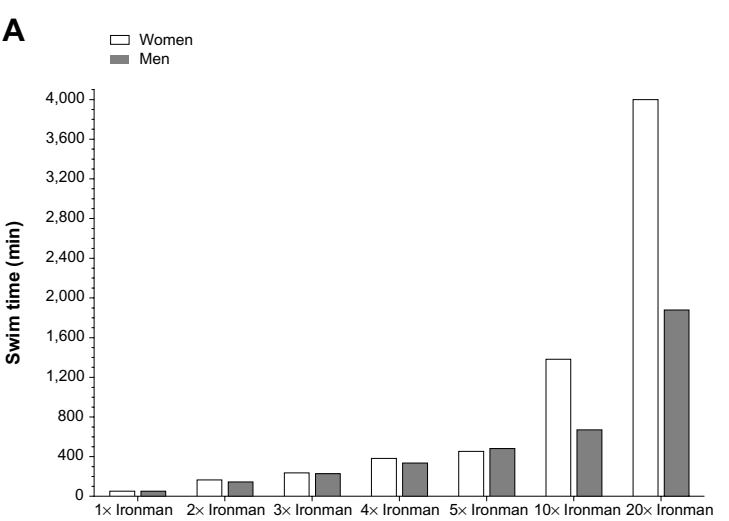

C

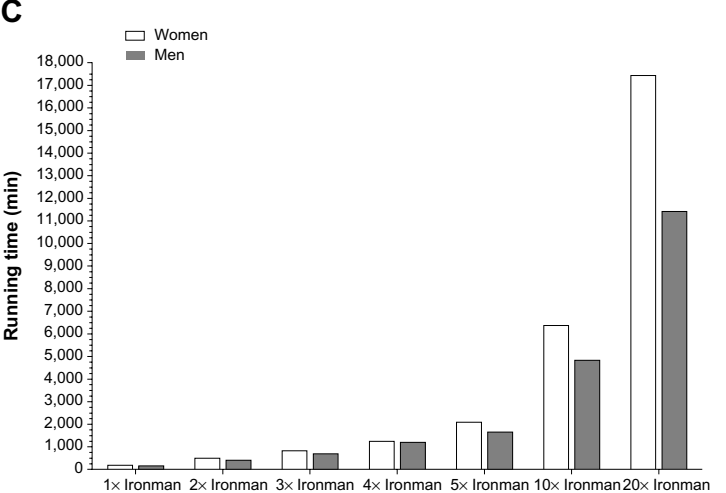

$\square$ Women

18,000

18,000
17,000

$16,000=$

15,000
14,000

13,000

政 $12,000=$

$11,000=$

10,000
9,000

을 8,000

$7,000=$

6,000
5,000

4,000

3,000 年

1,000

$0+\underset{1 \times 1 \text { Ironman }}{2 x}$

D

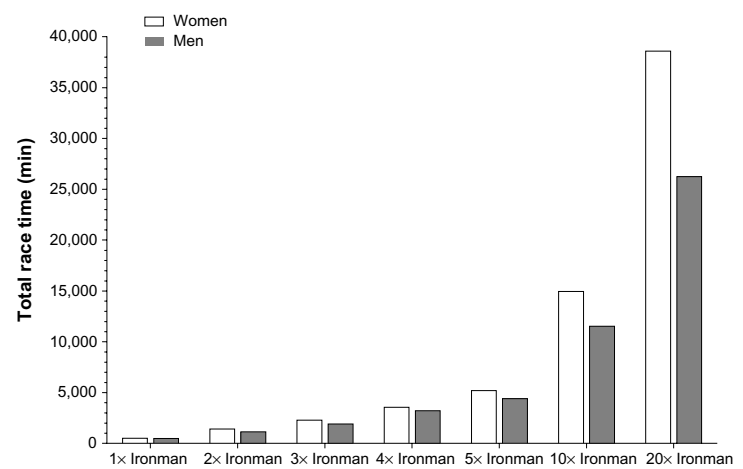

Figure I Split and overall race times of the fastest women and men ever.

Note: From left to right from Ironman Hawaii (IX Ironman) to double deca iron ultra-triathlon (20x Ironman).

A

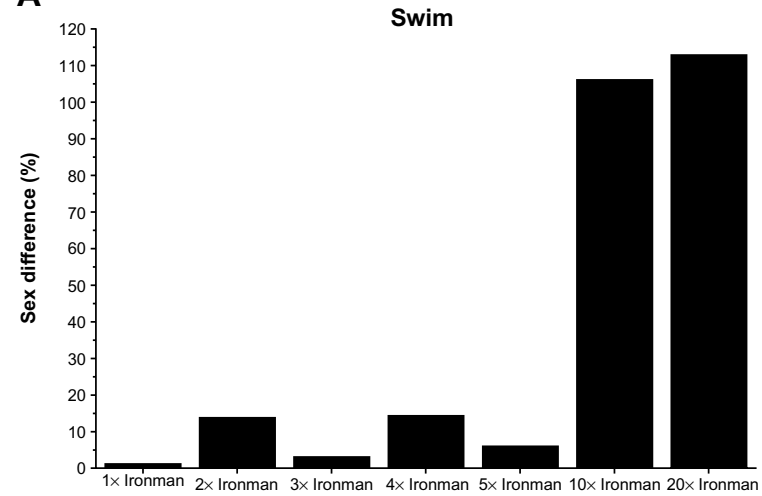

C

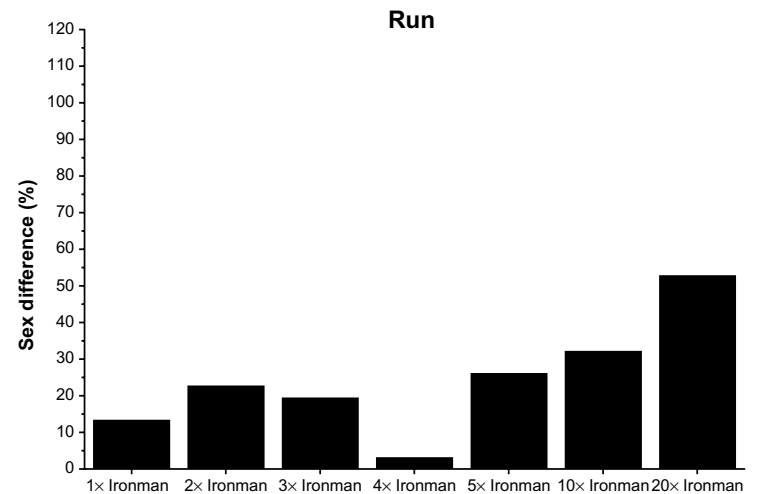

B

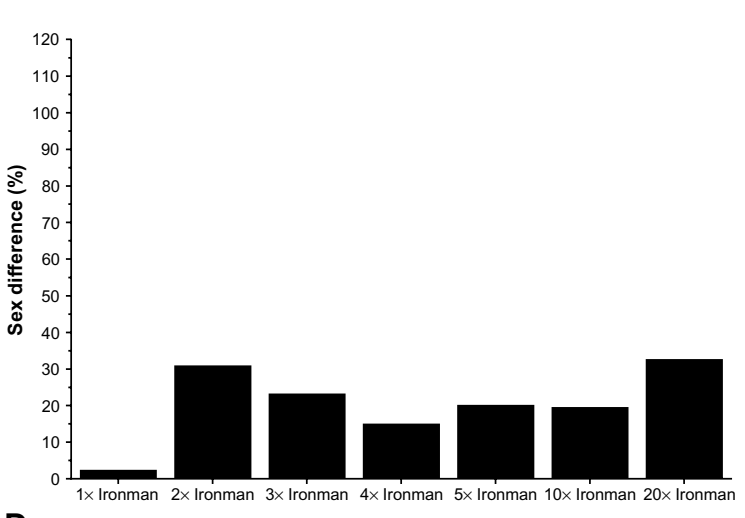

D

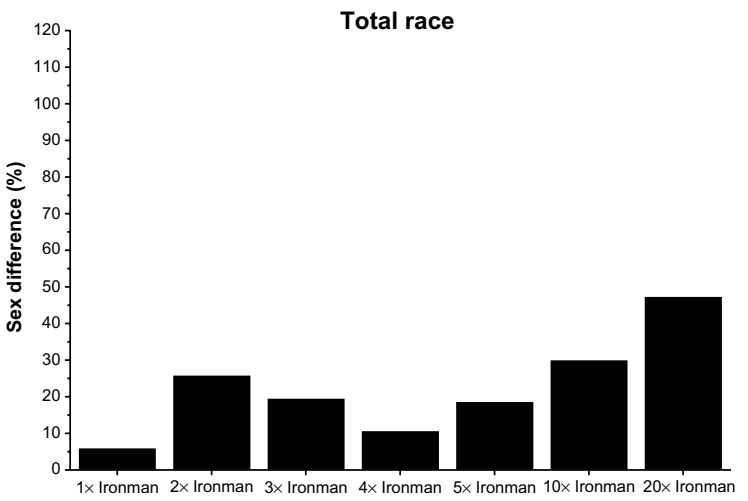

Figure 2 Sex difference in split and overall race times of the fastest women and men ever.

Note: From left to right from Ironman Hawaii (IX Ironman) to double deca iron ultra-triathlon (20× Ironman). 
differences for split (Figure 2A-C) and overall race times (Figure 2D) for the fastest finishers ever. The correlation analysis (Figure 3) showed an increase in the sex difference with increasing length of the race distance for the swimming split $\left(r^{2}=0.67, P=0.023\right)$ (Figure $\left.3 \mathrm{~A}\right)$, the running split $\left(r^{2}=0.77, P=0.009\right)$ (Figure 3B), and overall race times $\left(r^{2}=0.77, P=0.0087\right)$ (Figure 3D) but not for the cycling part $\left(r^{2}=0.26, P=0.23\right)$ (Figure 3C).

\section{Changes in performance across years}

Table 2 presents the results of the multilevel regression analyses for swimming, cycling, running, and overall race times. Swimming split times decreased nonlinearly in female (polynomial regression 3rd degree) and male (polynomial regression 7th degree) Ironman triathletes. From double iron to deca iron ultra-triathlon, no changes in the swimming split times were found across years. Cycling split times decreased nonlinearly in female and male ironman triathletes (polynomial regression 5th degree for both women and men). For the other triathlon distances, cycling split times decreased linearly in triple iron ultratriathlon for men, but no changes were found in the other race distances. Running split times decreased nonlinearly in female (polynomial regression 3rd degree) and male Ironman triathletes (polynomial regression 4th degree). For female triple iron ultra-triathletes, running split times increased linearly, whereas for male double iron ultra-triathletes, running split times decreased linearly. For female quintuple iron ultra-triathletes, running split times increased linearly across years. Overall race times decreased nonlinearly in female (polynomial regression 3rd degree) and male Ironman triathletes (polynomial regression 6th degree). In double iron ultra-triathlon, overall race times decreased nonlinearly in men (polynomial regression 4th degree). In triple iron ultra-triathlon, overall race times increased nonlinearly in women (polynomial regression 3rd degree). In quintuple iron ultra-triathlon, overall race times increased nonlinearly in men (polynomial regression 7 th degree). For the other races, overall race times remained unchanged across years.

\section{Changes in sex difference across years}

The sex difference in the swimming split time (Figure 4) decreased nonlinearly (Table 3) in Ironman (polynomial regression 5th degree) but was unchanged in all other
A

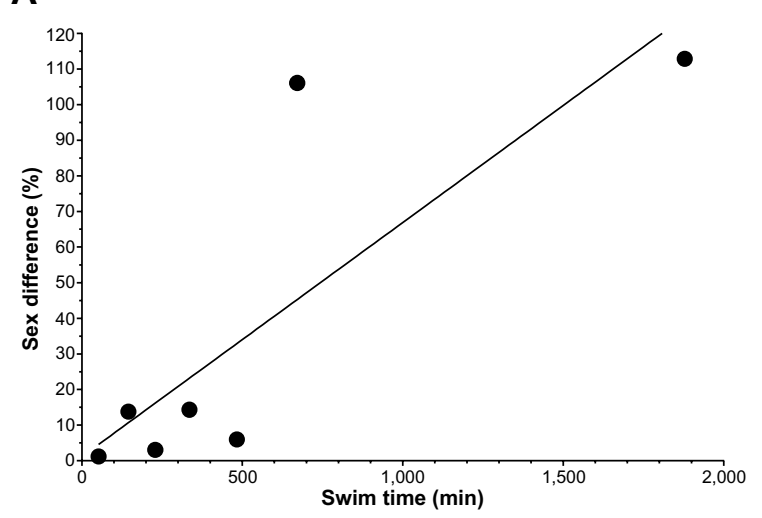

C

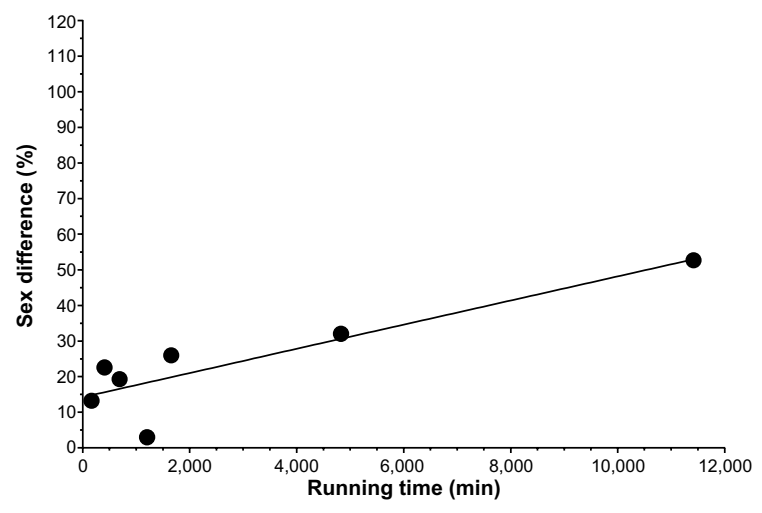

B

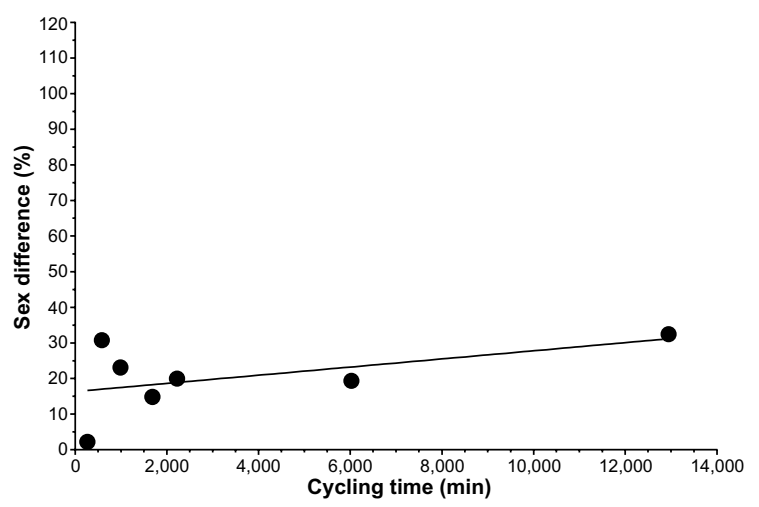

D

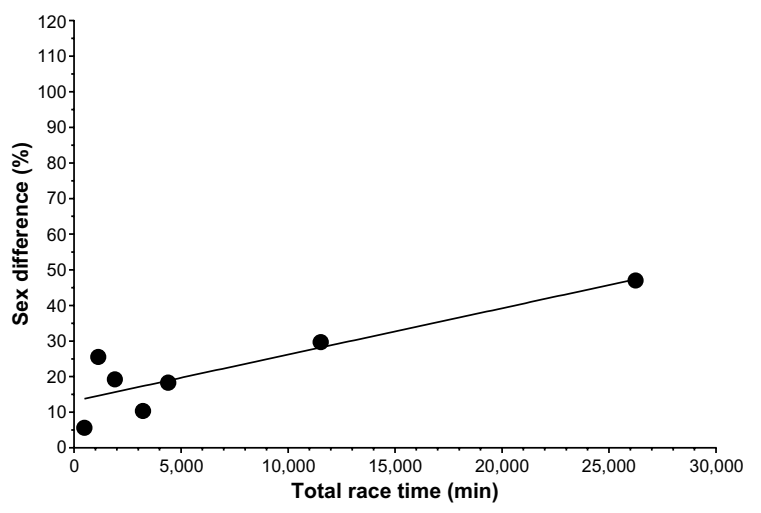

Figure 3 Sex difference in relation to split and overall race times for the fastest competitors ever across all distances.

Note: From left to right from Ironman Hawaii (IXIronman) to double deca iron ultra-triathlon (20× Ironman).

Abbreviation: min, minutes. 
Table 2 Multilevel regression analyses for changes across years in swimming, cycling, running, and overall race times with correction for multiple finishes

\begin{tabular}{|c|c|c|c|c|c|c|}
\hline & Sex & $\beta$ & SE $(\beta)$ & Stand $\beta$ & $\mathbf{T}$ & $P$-value \\
\hline \multicolumn{7}{|l|}{ Swimming } \\
\hline Ironman & Female & -0.26 & 0.08 & -0.46 & -3.01 & 0.005 \\
\hline Double iron & Female & -0.56 & 0.64 & -0.17 & -0.87 & 0.38 \\
\hline Triple iron & Female & -0.99 & 0.83 & -0.24 & -1.18 & 0.24 \\
\hline Quadruple iron & Female & -30.50 & $12.4 \mathrm{I}$ & -0.92 & -2.45 & 0.24 \\
\hline Quintuple iron & Female & -4.94 & 3.74 & -0.60 & -1.32 & 0.27 \\
\hline Deca iron & Female & -17.37 & 8.54 & -0.67 & -2.03 & 0.09 \\
\hline Ironman & Male & -0.25 & 0.09 & -0.42 & -2.73 & 0.01 \\
\hline Double iron & Male & 0.04 & 0.42 & 0.02 & 0.11 & 0.90 \\
\hline Triple iron & Male & 0.54 & 0.69 & 0.15 & 0.78 & 0.43 \\
\hline Quadruple iron & Male & -9.74 & 11.17 & -0.45 & -0.87 & 0.44 \\
\hline Quintuple iron & Male & 5.45 & 2.27 & 0.73 & 2.40 & 0.06 \\
\hline Deca iron & Male & 8.89 & 6.58 & 0.39 & 1.35 & 0.206 \\
\hline \multicolumn{7}{|l|}{ Cycling } \\
\hline Ironman & Female & -2.10 & 0.36 & $-0.7 \mathrm{I}$ & -5.79 & $<0.001$ \\
\hline Double iron & Female & -0.84 & 1.70 & -0.09 & -0.49 & 0.62 \\
\hline Triple iron & Female & 6.02 & 5.15 & 0.24 & 1.16 & 0.25 \\
\hline Quadruple iron & Female & -234.50 & 358.82 & -0.54 & -0.65 & 0.63 \\
\hline Quintuple iron & Female & 10.88 & 26.27 & 0.23 & $0.4 I$ & 0.70 \\
\hline Deca iron & Female & 133.97 & 120.79 & 0.44 & 1.10 & 0.31 \\
\hline Ironman & Male & -1.86 & 0.36 & -0.65 & -5.08 & $<0.001$ \\
\hline Double iron & Male & -1.54 & 0.81 & -0.34 & -1.88 & 0.07 \\
\hline Triple iron & Male & -4.59 & 1.72 & -0.47 & -2.66 & 0.01 \\
\hline Quadruple iron & Male & -36.63 & $|2.5|$ & -0.86 & -2.92 & 0.06 \\
\hline Quintuple iron & Male & $|8.4|$ & 8.80 & 0.68 & 2.09 & 0.09 \\
\hline Deca iron & Male & 62.44 & 40.72 & 0.43 & 1.53 & 0.15 \\
\hline \multicolumn{7}{|l|}{ Running } \\
\hline Ironman & Female & -1.75 & 0.32 & -0.68 & -5.38 & $<0.001$ \\
\hline Double iron & Female & -2.61 & 2.80 & -0.18 & -0.93 & 0.36 \\
\hline Triple iron & Female & 15.09 & 5.51 & 0.50 & 2.73 & 0.01 \\
\hline Quadruple iron & Female & -135.00 & 311.76 & -0.39 & -0.43 & 0.74 \\
\hline Quintuple iron & Female & 46.35 & 8.50 & 0.95 & 5.45 & 0.01 \\
\hline Deca iron & Female & 85.37 & 35.78 & 0.73 & 2.38 & 0.06 \\
\hline Ironman & Male & -0.93 & 0.20 & $-0.6 \mathrm{I}$ & -4.59 & $<0.001$ \\
\hline Double iron & Male & -3.25 & 1.04 & -0.51 & -3.12 & 0.004 \\
\hline Triple iron & Male & -0.83 & 2.31 & -0.07 & -0.35 & 0.72 \\
\hline Quadruple iron & Male & 8.68 & 37.83 & 0.13 & 0.23 & 0.83 \\
\hline Quintuple iron & Male & 43.76 & 21.54 & 0.67 & 2.03 & 0.09 \\
\hline Deca iron & Male & 3.47 & 48.80 & 0.02 & 0.07 & 0.94 \\
\hline \multicolumn{7}{|l|}{ Overall } \\
\hline Ironman & Female & -3.90 & 0.68 & -0.70 & -5.66 & $<0.001$ \\
\hline Double iron & Female & -3.66 & 4.03 & -0.17 & -0.90 & 0.37 \\
\hline Triple iron & Female & 20.23 & 9.29 & 0.42 & 2.17 & 0.04 \\
\hline Quadruple iron & Female & -401.00 & 682.42 & -0.50 & -0.58 & 0.66 \\
\hline Quintuple iron & Female & 55.49 & 39.25 & 0.63 & 1.41 & 0.25 \\
\hline Deca iron & Female & 201.96 & 117.33 & 0.61 & 1.72 & 0.14 \\
\hline Ironman & Male & -2.90 & 0.60 & -0.63 & -4.83 & $<0.001$ \\
\hline Double iron & Male & -4.54 & 1.65 & -0.46 & -2.75 & 0.01 \\
\hline Triple iron & Male & -4.93 & 3.12 & -0.30 & -1.58 & 0.12 \\
\hline Quadruple iron & Male & -37.74 & 26.53 & -0.63 & -1.42 & 0.25 \\
\hline Quintuple iron & Male & 69.53 & 25.85 & 0.76 & 2.69 & 0.04 \\
\hline Deca iron & Male & 78.95 & 67.18 & 0.34 & 1.17 & 0.26 \\
\hline
\end{tabular}

Abbreviations: $b$, regression coefficient; SE (b), standard error of regression coefficient; Stand $b$, standardized regression coefficient.

triathlon distances. In cycling (Figure 5), the sex difference increased linearly (Table 3 ) in triple iron ultra-triathlon but was unchanged in all other triathlon distances. The sex difference in the running split time (Figure 6) decreased nonlinearly (Table 3) in ironman (polynomial regression 9th degree) and increased linearly (Table 3) in triple iron ultra-triathlon but remained unchanged for all other triathlon distances. For overall race times (Figure 7), the 

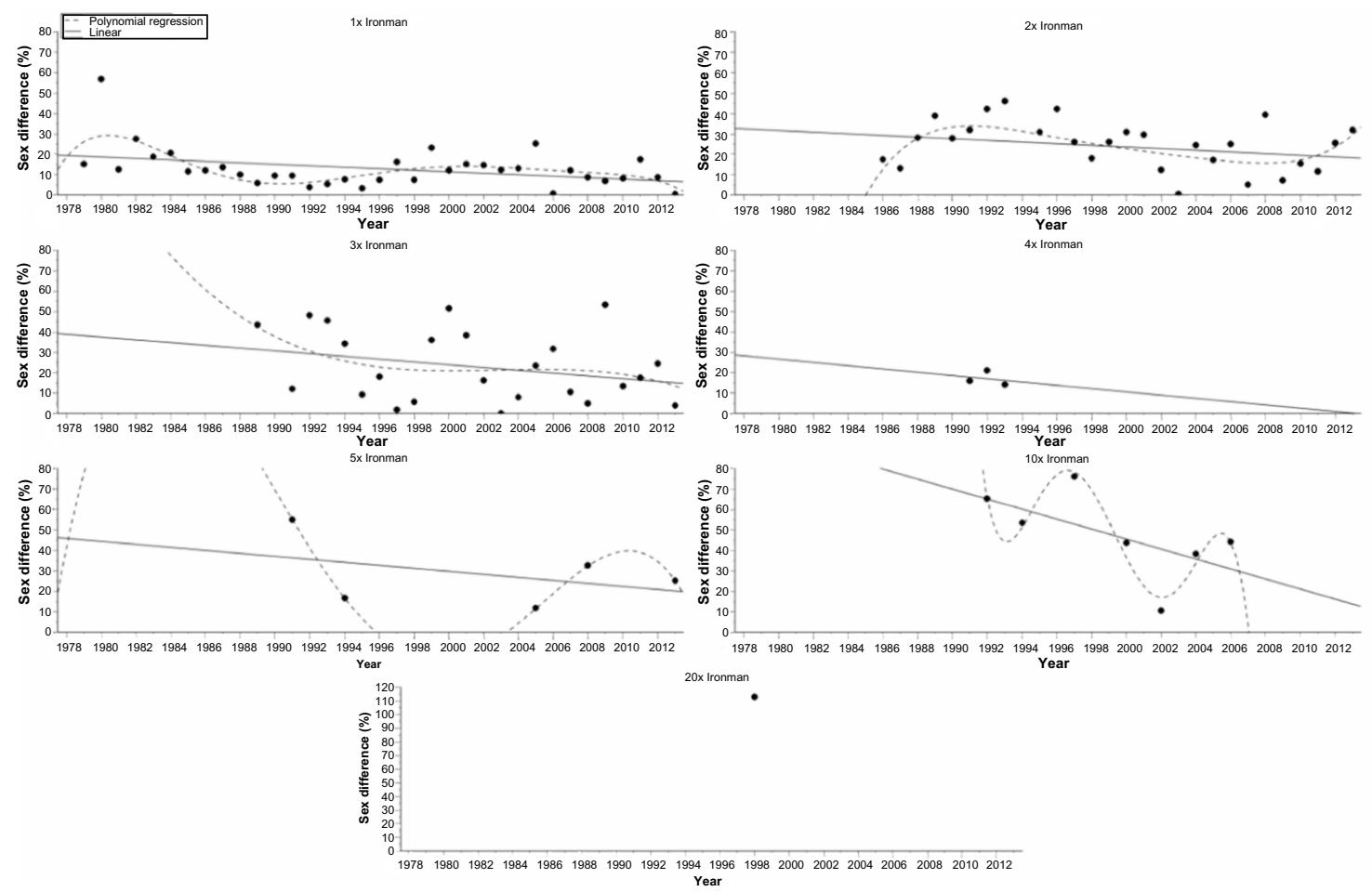

Figure 4 Sex difference in swimming for the annual fastest women and men from Ironman to double deca iron ultra-triathlon.

Note: Results are presented for the annual fastest for Ironman Hawaii (IXIronman), double iron ultra-triathlon ( $2 \times$ Ironman), triple iron ultra-triathlon ( $3 \times$ Ironman), quadruple

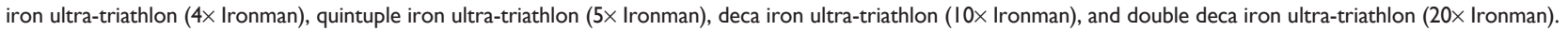

sex difference decreased nonlinearly (Table 3) in ironman (polynomial regression 4th degree) and increased nonlinearly (Table 3) (polynomial regression 5th degree) in triple iron ultra-triathlon.

\section{Discussion}

The intention of this study was to determine, firstly, whether the fastest women ever were faster than the fastest men ever, and secondly, to investigate potential changes in performance and sex difference in performance for the annual fastest finishers in race distances from Ironman to double deca iron ultra-triathlon. With the use of linear and nonlinear regression analyses, we intended to distinguish whether these athletes have reached their limits and whether women would be able to close the gap with men in the future. The most important findings were 1) the fastest men ever were faster than the fastest women ever, 2) the sex difference increased across race distances for the fastest finishers ever for swimming, running, and overall race time but not for cycling, 3) split and overall race times decreased nonlinearly across years in Ironman triathletes, and 4) the sex difference in performance decreased in Ironman and increased in triple iron ultra-triathlon but showed no changes across years for the other race distances.

\section{The fastest men ever were always faster than the fastest women ever}

A first important finding was that the fastest men ever were faster than the fastest women ever for split and overall race times, with the exception of the quintuple iron ultra-triathlon, where the fastest woman ever was faster in the swimming split than the fastest man ever. For ultra-swimming, women seemed to be able to achieve a similar or even better performance than men. Indeed, recent studies showed that women reduced the gap with men in ultra-distance swimming, ${ }^{17,18}$ and in an open-water ultra-swimming event, the Manhattan Island Marathon Swim, the fastest woman ever was faster than the fastest man ever. ${ }^{15}$ The distance of a $19 \mathrm{~km}$ ultra-swim in the quintuple iron ultra-triathlon might be an optimal race distance for female ultra-swimmers. The comparison of sex differences for the annual fastest elite ultra-swimmers competing in $5 \mathrm{~km}, 10 \mathrm{~km}$, and $25 \mathrm{~km}$ open-water ultra-distance swimming showed that the sex difference was lowest in $10 \mathrm{~km}$ ( $\sim 6.1 \%)$ compared to $5 \mathrm{~km}(\sim 7.6 \%)$ and $25 \mathrm{~km}(\sim 9.0 \%){ }^{16}$

\section{The sex difference in performance across race distances}

A second important finding was that the sex difference in performance increased with increasing length of the race 
Table 3 Multilevel regression analyses for changes across years in sex difference in swimming, cycling, running, and overall race times with correction for multiple finishes

\begin{tabular}{|c|c|c|c|c|c|}
\hline & $\beta$ & SE $(\beta)$ & Stand $\beta$ & $\mathbf{T}$ & $P$-value \\
\hline \multicolumn{6}{|l|}{ Swimming } \\
\hline Ironman & -0.36 & 0.15 & -0.37 & -2.31 & 0.02 \\
\hline Double iron & -0.40 & 0.28 & -0.26 & -1.42 & 0.16 \\
\hline Triple iron & -0.67 & 0.49 & -0.28 & -1.38 & 0.17 \\
\hline Quadruple iron & -0.80 & 3.48 & -0.22 & -0.22 & 0.85 \\
\hline Quintuple iron & -0.73 & 0.95 & -0.40 & -0.77 & 0.49 \\
\hline Deca iron & -2.43 & 1.43 & -0.60 & -1.69 & 0.15 \\
\hline \multicolumn{6}{|l|}{ Cycling } \\
\hline Ironman & -0.19 & 0.10 & -0.31 & -1.90 & 0.06 \\
\hline Double iron & 0.03 & 0.28 & 0.02 & 0.11 & 0.90 \\
\hline Triple iron & 0.94 & 0.32 & 0.52 & 2.88 & 0.00 \\
\hline Quadruple iron & -10.39 & 23.03 & $-0.4 I$ & -0.45 & 0.73 \\
\hline Quintuple iron & -0.11 & 1.72 & -0.03 & -0.06 & 0.95 \\
\hline Deca iron & 0.06 & 1.17 & 0.02 & 0.05 & 0.95 \\
\hline \multicolumn{6}{|l|}{ Running } \\
\hline Ironman & -0.43 & 0.09 & -0.63 & -4.68 & $<0.001$ \\
\hline Double iron & 0.22 & 0.50 & 0.08 & 0.45 & 0.65 \\
\hline Triple iron & 1.67 & $0.5 \mathrm{I}$ & 0.57 & 3.27 & 0.003 \\
\hline Quadruple iron & -6.10 & $|3.8|$ & -0.40 & -0.44 & 0.73 \\
\hline Quintuple iron & 0.47 & 1.59 & 0.17 & 0.30 & 0.78 \\
\hline Deca iron & 4.09 & 2.53 & 0.58 & 1.61 & 0.16 \\
\hline \multicolumn{6}{|l|}{ Overall } \\
\hline Ironman & -0.26 & 0.05 & -0.63 & -4.68 & $<0.001$ \\
\hline Double iron & 0.01 & 0.30 & 0.009 & 0.04 & 0.96 \\
\hline Triple iron & I.II & 0.31 & 0.6 & 3.58 & 0.002 \\
\hline Quadruple iron & -7.68 & 16.91 & $-0.4 I$ & -0.45 & 0.72 \\
\hline Quadruple iron & -0.20 & 1.58 & -0.07 & -0.13 & 0.9 \\
\hline Deca iron & 1.63 & 0.81 & 0.66 & 2.0 & 0.1 \\
\hline
\end{tabular}

Abbreviations: $b$, regression coefficient; $\mathrm{SE}(b)$, standard error of regression coefficient; Stand $b$, standardized regression coefficient.

distance for swimming, running, and overall race times but not for cycling. The sex differences were $\sim 20 \%$ for both split and overall race times for the shorter ultra-triathlon distances (from Ironman to quadruple iron ultra-triathlon distance) but increased to higher values in the longer ultra-triathlon distances (from quintuple to double deca iron ultratriathlon distance). Sex differences of $\sim 20 \%$ are common in ultra-endurance performances such as in ultra-running ${ }^{12}$ and in ultra-cycling ${ }^{13}$ but not in ultra-swimming..$^{15} \mathrm{~A}$ potential explanation for the high sex differences in the longer ultratriathlon distances compared to the single sports might be the "history" of the triathlete. Triathletes have to compete in three different disciplines, and the background might be different for each athlete. Athletes may start competing in triathlons as a former swimmer, cyclist, runner, or they may even come from other sport disciplines. Additionally, cycling and running performance are the most important disciplines in an ultra-triathlon, ${ }^{21}$ and the contribution of swimming to overall ultra-triathlon performance was lower than for cycling and running. ${ }^{22}$ Since cycling and running performance are highly predictive for ultra-triathlon performance, ${ }^{21,22}$ the history of an ultra-triathlete might be running or cycling rather than swimming.

In recent years, it has been assumed that women would be able to outperform men in ultra-endurance performances such as ultra-marathon running. Beneke et al reported that men were faster than women in the $216 \mathrm{~km}$ Badwater ultra-marathon during the 1980s and 1990s. ${ }^{23}$ However, in 2002 and 2003, a woman was able to beat the fastest man by $\sim 4.5$ hours and $\sim 0.5$ hour, respectively. ${ }^{24}$ Furthermore, since 2002, up to three women finished the Badwater race within the top five overall, although more men than women competed. ${ }^{24} \mathrm{~A}$ similar finding of a female runner beating all men has been reported for a 45 -year-old female ultra-marathoner winning the Deutschlandlauf, a 17-day multistage ultra-marathon covering a total distance of $1,200 \mathrm{~km}$ from the north to the south of Germany. ${ }^{25}$ The woman won the race $\sim 8$ hours ahead of the fastest man. ${ }^{25}$ The assumption that women might be able to outrun men in ultra-marathon running was supported by the comparison of female and male performance in different ultra-running distances. Hoffman showed that women and men who were matched for $50 \mathrm{~km}$ trail running performance also performed similarly in trail runs of $80 \mathrm{~km}$ and $161 \mathrm{~km}$ distances. ${ }^{26}$ Physiological assumptions to explain why women could outrun men included differences in the ability to run at a higher percentage of maximum oxygen uptake $\left(\mathrm{VO}_{2} \max \right),{ }^{27}$ differences in the use of ingested and stored glycogen, ${ }^{27}$ and differences in lipid metabolism. ${ }^{28}$ During long-duration exercise performed at low to moderate intensities, women showed a higher lipid utilization and a lower carbohydrate and protein metabolism compared to equally trained and nourished men. ${ }^{29}$ In addition, women used more fat stored in the legs compared to men during performance. ${ }^{30}$ Coast et al, at the end of 2002, compared the world best running performances in race distances from $100 \mathrm{~m}$ to $200 \mathrm{~km} .{ }^{31}$ Men were on average $\sim 12.4 \%$ faster than women. There was a significant increase in the sex difference in running speed across race distances. Also for ultra-triathlons, the sex difference increased with increasing race distance for swimming, running, and overall race times. It seems impossible that women could outperform men in ultra-triathlons as presented by Coast et al for running. ${ }^{31}$ Potential reasons to explain why men are faster than women in endurance performance are the higher power in upper and lower limbs in men compared to women. ${ }^{32,33}$ 

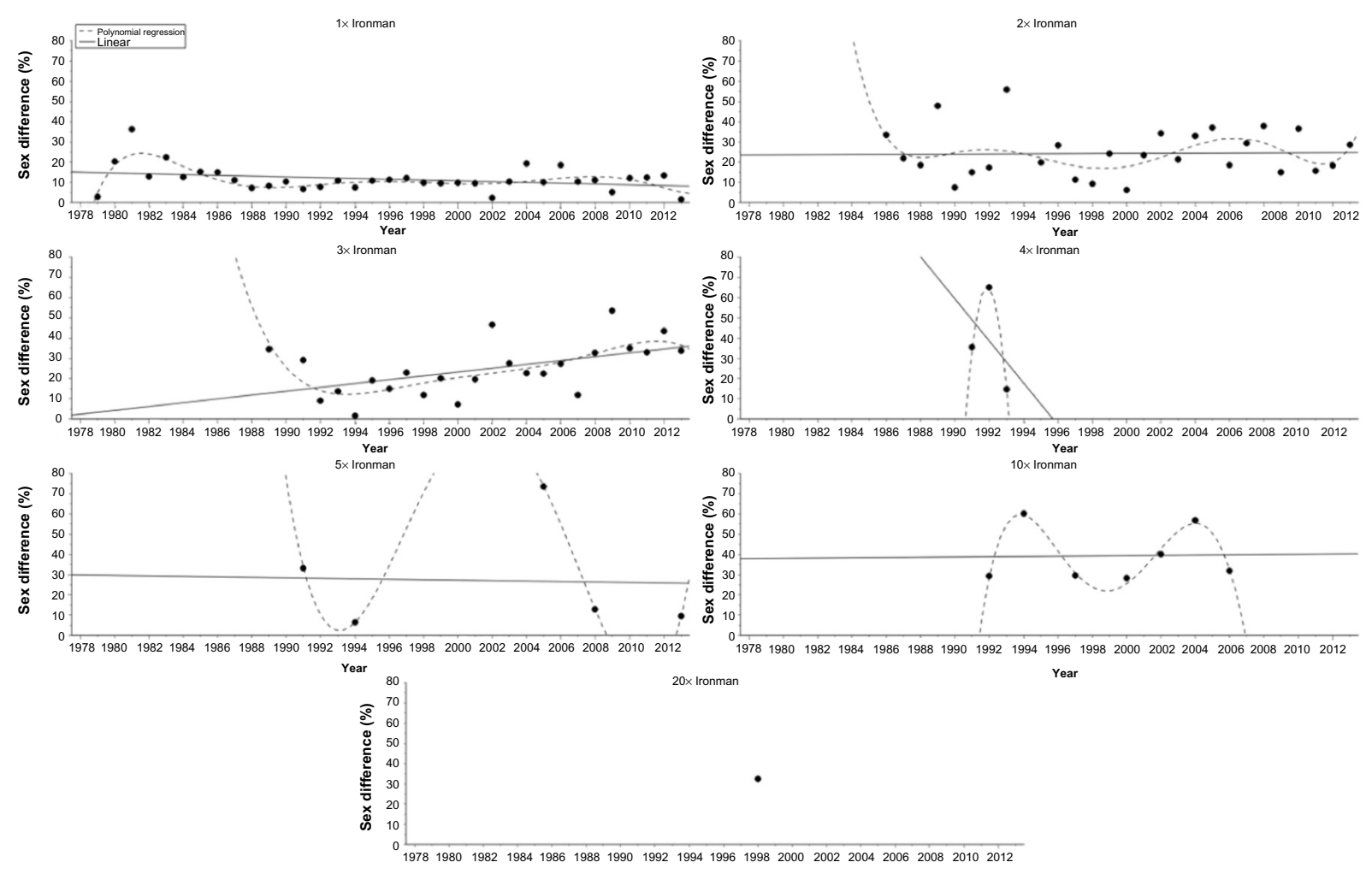

Year

Figure 5 Sex difference in cycling for the annual fastest women and men from Ironman to double deca iron ultra-triathlon.

Note: Results are presented for the annual fastest for Ironman Hawaii (I $\times$ Ironman), double iron ultra-triathlon ( $2 \times$ Ironman), triple iron ultra-triathlon ( $3 \times$ Ironman), quadruple iron ultra-triathlon ( $4 \times$ Ironman), quintuple iron ultra-triathlon ( $5 \times$ Ironman), deca iron ultra-triathlon (I0× Ironman), and double deca iron ultra-triathlon $(20 \times$ Ironman).

\section{Changes in split and overall race times across years}

The annual fastest women and men improved split and overall race times nonlinearly in Ironman Hawaii. Although Ironman triathletes still improve in Ironman Hawaii, ${ }^{34}$ and the actual course records were set recently for men in 2011 by Craig Alexander in 8:03:56 hours:minutes:seconds and for women in 2013 by Mirinda Carfrae in 8:52:14 hours:minutes:seconds, the nonlinear decrease in split and overall race times suggests that both women and men have reached their limits in Ironman Hawaii. Also for the longer triathlon distances, the fastest athletes seem to have reached their limits in performance. In the double iron ultra-triathlon, running times decreased linearly, and overall race times decreased nonlinearly in men. Although men improved in running, the nonlinear decrease in overall race times suggests that men have reached their limits also in the double iron ultra-triathlon. In the triple iron ultra-triathlon, cycling split times decreased in men, and running split times and overall race times increased in women. The unchanged race times in men and the increase in overall race times in women suggest that athletes have also reached their limits in the triple iron ultra-triathlon. For the longer distances, performance showed no changes across years and, therefore, no further improvements should be expected in the near future in race distances longer than the triple iron ultra-triathlon.

\section{Changes in sex differences across years}

In Ironman Hawaii, men were faster than women, but the sex difference decreased nonlinearly in swimming, running, and overall race time. For the other race distances, however, the sex difference increased in cycling, running, and overall race time in the triple iron ultra-triathlon. For longer distances, the sex difference showed no changes across time. The sex difference in performance between female and male endurance athletes might be partially explained by anthropometric differences between women and men, such as differences in skeletal muscle mass and body fat. ${ }^{35,39}$ Knechtle et al argued that the higher sex difference in ultra-endurance ultratriathlon races was most probably due to the lower skeletal muscle mass in women compared to men. ${ }^{35}$ It has been shown that male ultra-endurance athletes had a higher skeletal 

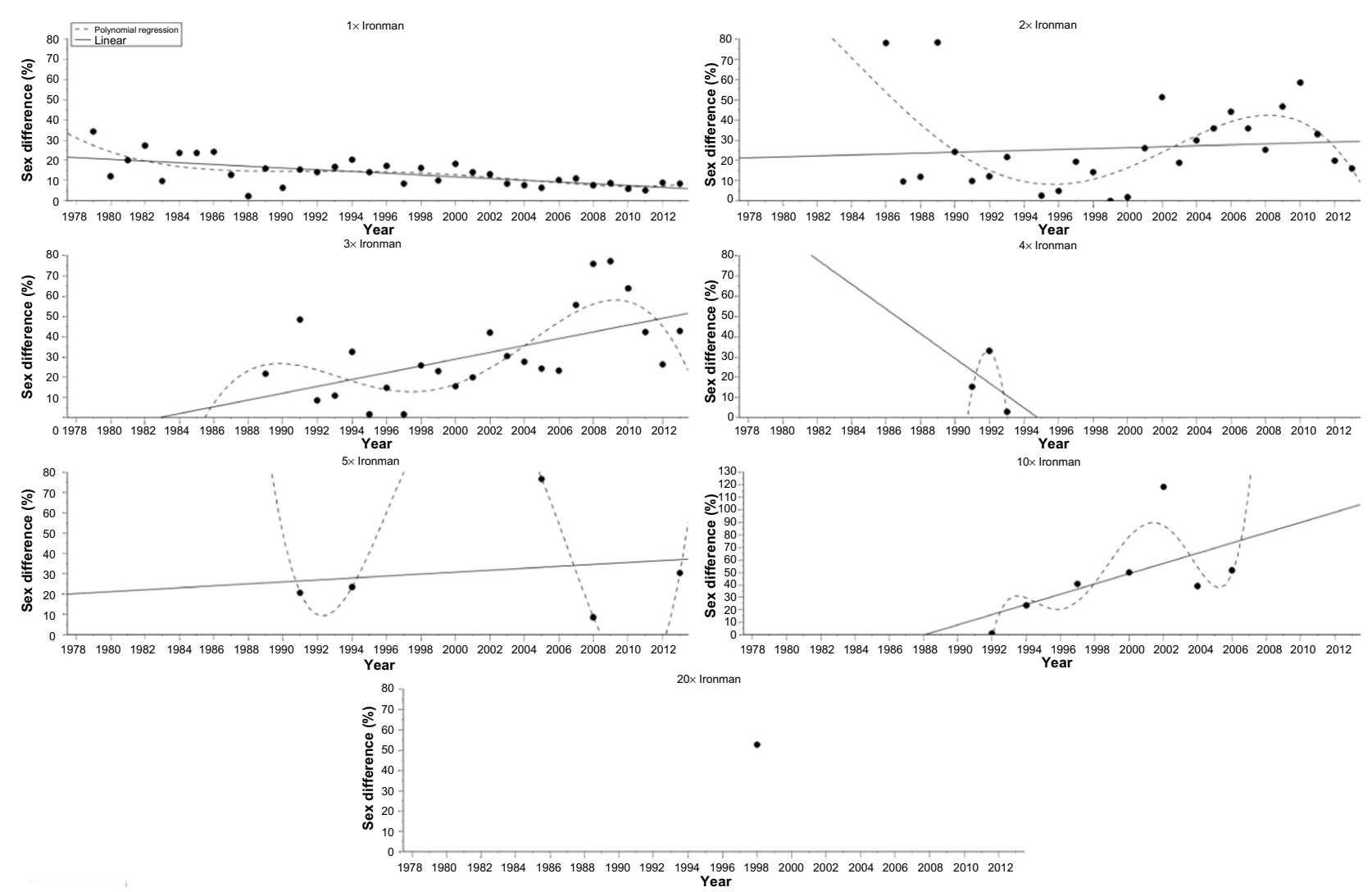

Figure 6 Sex difference in running for the annual fastest women and men from Ironman to double deca iron ultra-triathlon.

Note: Results are presented for the annual fastest for Ironman Hawaii (I $\times$ Ironman), double iron ultra-triathlon ( $2 \times$ Ironman), triple iron ultra-triathlon $(3 \times$ Ironman), quadruple

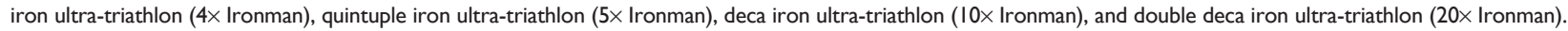
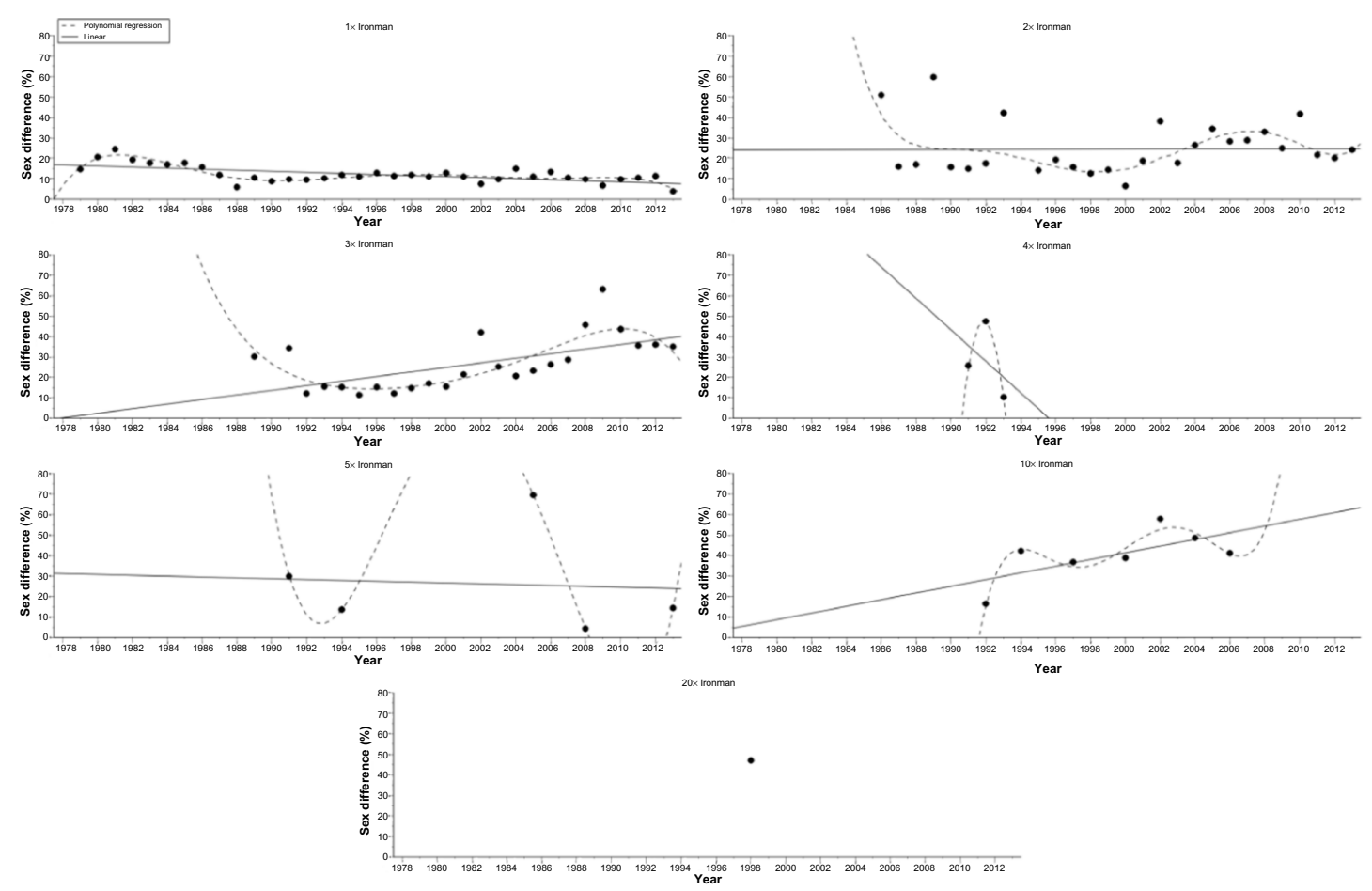

Figure 7 Sex difference in overall race time for the annual fastest women and men from Ironman to double deca iron ultra-triathlon.

Note: Results are presented for the annual fastest for Ironman Hawaii (I $\times$ Ironman), double iron ultra-triathlon ( $2 \times$ Ironman), triple iron ultra-triathlon ( $3 \times$ Ironman), quadruple

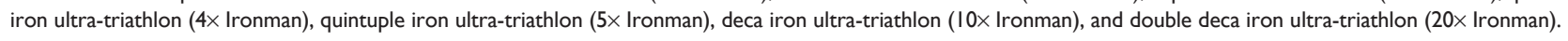


muscle mass than female ultra-endurance athletes. ${ }^{36-39}$ Male Ironman triathletes had a $\sim 46 \%$ higher muscle mass compared to female Ironman triathletes ${ }^{36}$ For ultra-runners, male ultra-runners had a $\sim 38 \%$ higher muscle mass compared to female ultra-runners. ${ }^{37,38}$ Comparing ultra-runners and ultraswimmers, the sex difference in skeletal muscle mass was considerably higher in ultra-runners. ${ }^{39}$ Male open-water ultraswimmers had $\sim 45 \%$ more muscle mass compared to female open-water ultra-swimmers. ${ }^{39}$ These differences in skeletal muscle mass between female and male ultra-endurance athletes might explain the increase in sex difference with increasing length of an endurance performance. Men with a higher muscle mass might have more reserve for longer endurance than women. A further aspect is the fact that cycling performance is related to leg power. ${ }^{40}$ It has been shown that ultra-endurance performance leads to a decrease in lean body mass. ${ }^{41}$ Since women have a lower skeletal muscle mass, a prolonged cycling performance might lead to a higher relative decrease in lower leg skeletal muscle mass in women compared to men; therefore, cycling performance might become more reduced in women compared to men. Although the sex difference in cycling split performance showed no change across race distances for the fastest finishers ever, women were slower in running, where the sex difference increased with increasing race distance. Another important anthropometric predictor variable for a successful outcome in endurance competitions is body fat. It has been shown in male triathletes and male ultra-marathoners that lower body fat was associated with faster race times. ${ }^{42,43}$ Mean body fat percentages for recreational male and female Ironman triathletes were $\sim 14 \%$ and $\sim 23 \%$, respectively. ${ }^{36}$ Therefore, male athletes with lower body fat seemed to have an advantage in running and maybe also in cycling compared to female athletes. Regarding swimming, however, body fat seems to have a different effect. Female swimmers had proportionately more fatty tissue located caudally than male swimmers. ${ }^{44}$ As recent studies showed that women were able to achieve a similar performance as men in ultra-distance swimming, ${ }^{45,46}$ a higher body fat could give female ultra-swimmers an advantage, such as improved buoyancy. Most probably, the higher body fat in women is the main reason that female openwater ultra-swimmers were able to beat male open-water ultra-swimmers in the Manhattan Island marathon swim. ${ }^{15}$ However, overall race times in triathlons mainly depend on cycling and running. ${ }^{21,47}$ Therefore, the higher skeletal muscle mass and the lower body fat in men may support the theory of a biologically-based performance difference between female and male ultra-endurance athletes.

\section{Low female participation in ultra-endurance triathlon}

Female participation was highest in Ironman Hawaii and lowest in deca iron ultra-triathlon. In double deca iron ultra-triathlon, only one woman ever finished. The low number of women competing in the very long ultra-distance triathlons (longer than triple iron ultra-triathlon) might considerably influence both performance and sex difference in performance. There are considerably more Ironman races offered around the world compared to ultra-triathlons longer than the Ironman..$^{20,48}$ This large difference in race opportunity might influence the participation trends. Additionally, the top athletes in an Ironman race can earn prize money. For example, a total of $\$ 125,000$ US will be distributed as prize money among the eight fastest women and men in Ironman Germany, where women and men will get the same amount. ${ }^{49}$ In ultra-triathlons longer than an Ironman, generally no prize money is awarded..$^{20}$

The highest percentage for female finishers was in Ironman Hawaii, which has been held annually since $1978 .{ }^{50}$ Athletes must qualify for Ironman Hawaii since the number of slots for female and male competitors is limited. ${ }^{51,52}$ Ironman Hawaii takes place every October in Kailua-Kona, Hawaii, and in addition to the 1,800 professional and age-group qualifiers, 100 legacy and 100 lottery athletes compete in this event. ${ }^{52}$ Although double and triple iron ultra-triathlons have been held since 1985 and 1988, respectively, and athletes are not required to qualify, ${ }^{20}$ the number of female and male finishers is considerably lower compared to Ironman Hawaii. Additionally, the number of competitors in a double or a triple iron ultra-triathlon race is far below $100 .{ }^{20}$ Double and triple iron ultra-triathlons are held annually, but ultra-triathlons longer than the triple iron are not held regularly. ${ }^{20}$ Indeed, only two races were ever held for double deca iron ultratriathlon where only one woman ever successfully finished. Most probably, the decrease in number of female finishers with increasing length of the ultra-triathlon races might explain the finding that sex difference was unchanged across race distances.

Female participation is also low in other endurance disciplines such as running. Coast et al compared the world best running performances from $100 \mathrm{~m}$ to $200 \mathrm{~km},{ }^{31}$ and men were faster than women with an average sex difference of $\sim 12.4 \%$ and longer running distances were associated with higher sex differences. The authors argued that their results might be confounded by the lower number of women in longer running distance events. Indeed, men are overrepresented in sports, and the faster performance in men compared to women is 
most probably due to men's greater training motivation. ${ }^{53}$ Popular and modern male sports require the skills needed for success in male-male physical competition, whereas female ultra-endurance athletes are rather task-oriented, internally motivated, healthy, and financially conscious individuals. ${ }^{54,55}$

A further explanation for the sex difference in both participation and performance in ultra-endurance triathlons could be a different motivation between women and men to compete in these ultra-endurance races. Personality, motivation, and goal orientation have been investigated in different sports disciplines. ${ }^{56,57}$ Women were motivated to exercise regularly to reduce body fat, to increase physical fitness, or to improve social interactions. ${ }^{56-60}$ The aspect of competing and winning a race seemed to be of lower importance for women compared to men. For example, the aspect of social affiliation and improving physical fitness was more important for female marathoners than achievements and personal accomplishments. ${ }^{61,62}$ For female ultra-marathoners, Krouse et al investigated the influence of motivation, goal orientation, and training on performance, where general health orientation and psychological coping were the strongest motivational factors. ${ }^{55}$

\section{Limitations}

Limitations of this study are that aspects such as training of the subjects, ${ }^{63,64}$ previous experience, ${ }^{63}$ anthropometric characteristics, ${ }^{36,64}$ age $, 34,65,66$ nutrition, ${ }^{67}$ pacing strategy, ${ }^{68}$ nationality, ${ }^{69,70}$ and environmental conditions ${ }^{71}$ were not considered. These characteristics might have had an influence on the performance of the athletes.

\section{Conclusion}

Although women reduced the sex difference in the shorter ultra-triathlon distances (ie, Ironman distance) across years, the sex difference in longer triathlon distances (double and the triple iron ultra-triathlon) remained greater compared to the single Ironman distance. The fastest men ever were faster than the fastest women ever, and the sex difference increased with increasing length of the race distance for swimming, running, and overall race times, but not for cycling. In summary, although women reduced the sex difference nonlinearly in shorter ultra-triathlon distances (Ironman distance), the sex difference increased or remained unchanged across years for longer distances (longer than the Ironman distance). It seems very unlikely that female top performers will ever outrun male top performers in ultratriathlons in the near future.

\section{Disclosure}

The authors report no conflicts of interest in this work.

\section{References}

1. Bentley DJ, Millet GP, Vleck VE, McNaughton LR. Specific aspects of contemporary triathlon: implications for physiological analysis and performance. Sports Med. 2002;32(6):345-359.

2. Stevenson JL, Song H, Cooper JA. Age and sex differences pertaining to modes of locomotion in triathlon. Med Sci Sports Exerc. 2013;45(5):976-984.

3. Lenherr R, Knechtle B, Rüst CA, Rosemann T, Lepers R. From double iron to double deca iron ultra-triathlon - a retrospective data analysis from 1985 to 2011. Physical Culture and Sport. Studies and Research. 2012;54(1):55-67.

4. Lepers R, Maffiuletti NA. Age and gender interactions in ultraendurance performance: insight from the triathlon. Med Sci Sports Exerc. 2011;43(1):134-139.

5. Lepers R. Analysis of Hawaii ironman performances in elite triathletes from 1981 to 2007. Med Sci Sports Exerc. 2008;40(10):1828-1834.

6. Rüst CA, Lepers R, Stiefel M, Rosemann T, Knechtle B. Performance in Olympic triathlon: changes in performance of elite female and male triathletes in the ITU World Triathlon Series from 2009 to 2012. Springerplus. 2013;2:685.

7. Rüst CA, Knechtle B, Rosemann T, Lepers R. Sex difference in race performance and age of peak performance in the Ironman Triathlon World Championship from 1983 to 2012. Extrem Physiol Med. 2012;1(1):15.

8. Sigg K, Knechtle B, Rüst CA, Knechtle P, Lepers R, Rosemann T. Sex difference in Double Iron ultra-triathlon performance. Extrem Physiol Med. 2013;2(1):12.

9. Rüst CA, Knechtle B, Knechtle P, Rosemann T, Lepers R. Participation and performance trends in triple iron ultra-triathlon - a cross-sectional and longitudinal data analysis. Asian J Sports Med. 2012;3(3): $145-152$.

10. Reinboud W. Linear models can't keep up with sport gender gap. Nature. 2004;432(7014):147; author reply 147.

11. Peter L, Rüst CA, Knechtle B, Rosemann T, Lepers R. Sex differences in 24-hour ultra-marathon performance - a retrospective data analysis from 1977 to 2012. Clinics (Sao Paulo). 2014;69(1):38-46.

12. Zingg MA, Karner-Rezek K, Rosemann T, Knechtle B, Lepers R, Rüst CA. Will women outrun men in ultra-marathon road races from $50 \mathrm{~km}$ to 1,000 km? Springerplus. 2014;3:97.

13. Rüst CA, Knechtle B, Rosemann T, Lepers R. Men cross America faster than women - the "Race Across America" from 1982 to 2012. Int J Sports Physiol Perform. 2013;8(6):611-617.

14. Zingg M, Knechtle B, Rüst CA, Rosemann T, Lepers R. Age and gender difference in non-drafting ultra-endurance cycling performance - the 'Swiss Cycling Marathon'. Extrem Physiol Med. 2013;2(1):18.

15. Knechtle B, Rosemann T, Lepers R, Rüst CA. Women outperform men in ultra-distance swimming - the 'Manhattan Island Marathon Swim' from 1983 to 2013. Int J Sports Physiol Perform. Epub February 28, 2014.

16. Zingg MA, Rüst CA, Rosemann T, Lepers R, Knechtle B. Analysis of sex differences in open-water ultra-distance swimming performances in the FINA World Cup races in $5 \mathrm{~km}, 10 \mathrm{~km}$ and $25 \mathrm{~km}$ from 2000 to 2012. BMC Sports Sci Med Rehabil. 2014;6(1):7.

17. Rüst CA, Lepers R, Rosemann T, Knechtle B. Will women soon outperform men in open-water ultra-distance swimming in the 'Maratona del Golfo Capri-Napoli’? Springerplus. 2014;3:86.

18. Rüst CA, Knechtle B, Rosemann T, Lepers R. Women reduced the sex difference in open-water ultra-distance swimming [Formula: see text] La Traversée Internationale du Lac St-Jean, 1955-2012. Appl Physiol Nutr Metab. 2014;39(2):270-273.

19. Ironman World Championship http://www.ironmanworldchampionship. com.

20. International Ultra -Triathlon Association (IUTA) http://www.iutasport.com. 
21. Knechtle B, Duff B, Amtmann G, Kohler G. Cycling and running performance, not anthropometric factors, are associated with race performance in a triple iron triathlon. Res Sports Med. 2007;15(4):257-269.

22. Lepers R, Knechtle B, Knechtle P, Rosemann T. Analysis of ultratriathlon performances. Open Access J Sports Med. 2011;2:131-136.

23. Beneke R, Leithäuser RM, Doppelmayr M. Women will do it in the long run. Br J Sports Med. 2005;39(7):410.

24. Badwater. Badwater Ultramarathon Race Results, 2000 to the present [webpage on the Internet]. Available from: http://www.badwater.com/ results/index.html. Accessed January 12, 2014.

25. Knechtle B, Duff B, Schulze I, Kohler G. The effects of running 1,200 km within 17 days on body composition in a female ultrarunnerDeutschlandlauf 2007. Res Sports Med. 2008;16(3):167-188.

26. Hoffman MD. Ultramarathon trail running comparison of performance-matched men and women. Med Sci Sports Exerc. 2008;40(9):1681-1686.

27. Riddell MC, Partington SL, Stupka N, Armstrong D, Rennie C, Tarnopolsky MA. Substrate utilization during exercise performed with and without glucose ingestion in female and male endurance trained athletes. Int J Sport Nutr Exerc Metab. 2003;13(4): 407-421.

28. Perreault L, Lavely JM, Kittelson JM, Horton TJ. Gender differences in lipoprotein lipase activity after acute exercise. Obes Res. 2004;12(2) 241-249.

29. Tarnopolsky LJ, MacDougall JD, Atkinson SA, Tarnopolsky MA, Sutton JR. Gender differences in substrate for endurance exercise. J Appl Physiol (1985). 1990;68(1):302-308.

30. Roepstorff C, Steffensen CH, Madsen M, et al. Gender differences in substrate utilization during submaximal exercise in endurancetrained subjects. Am J Physiol Endocrinol Metab. 2002;282(2): E435-E447.

31. Coast JR, Blevins JS, Wilson BA. Do gender differences in running performance disappear with distance? Can J Appl Physiol. 2004;29(2): 139-145.

32. Nikolaidis PT. Age- and sex-related differences in force-velocity characteristics of upper and lower limbs of competitive adolescent swimmers. J Hum Kinet. 2012;32:87-95.

33. Nikolaidis P. Gender differences in anaerobic power in physical education and sport science students. J Phys Ed Sport. 2009;24(3):140-145.

34. Gallmann D, Knechtle B, Rüst CA, Rosemann T, Lepers R. Elite triathletes in 'Ironman Hawaii' get older but faster. Age (Dordr). 2014;36(1): 407-416.

35. Knechtle B, Knechtle P, Lepers R. Participation and performance trends in ultra-triathlons from 1985 to 2009. Scand J Med Sci Sports. 2011;21(6):e82-e90.

36. Knechtle B, Wirth A, Baumann B, Knechtle P, Rosemann T, Oliver S. Differential correlations between anthropometry, training volume, and performance in male and female Ironman triathletes. J Strength Cond Res. 2010;24(10):2785-2793.

37. Knechtle B, Senn O, Imoberdorf R, et al. Maintained total body water content and serum sodium concentrations despite body mass loss in female ultra-runners drinking ad libitum during a $100 \mathrm{~km}$ race. Asia Pac J Clin Nutr. 2010;19(1):83-90.

38. Knechtle B, Senn O, Imoberdorf R, et al. No fluid overload in male ultra-runners during a $100 \mathrm{~km}$ ultra-run. Res Sports Med. 2011;19(1): $14-27$.

39. Weitkunat T, Knechtle B, Knechtle P, Rüst CA, Rosemann T. Body composition and hydration status changes in male and female openwater swimmers during an ultra-endurance event. J Sports Sci. 2012;30(10):1003-1013.

40. Hopker J, Jobson S, Carter H, Passfield L. Cycling efficiency in trained male and female competitive cyclists. $J$ Sports Sci Med. 2010;9(2): 332-337.

41. Schütz UH, Billich C, König K, et al. Characteristics, changes and influence of body composition during a $4486 \mathrm{~km}$ transcontinental ultramarathon: results from the TransEurope FootRace mobile whole body MRI-project. BMC Med. 2013;11:122.
42. Knechtle B, Knechtle P, Rüst CA, Rosemann T. A comparison of anthropometric and training characteristics of Ironman triathletes and triple iron ultra-triathletes. J Sports Sci. 2011;29(13):1373-1380.

43. Rüst CA, Knechtle B, Knechtle P, Rosemann T. Similarities and differences in anthropometry and training between recreational male 100-km ultra-marathoners and marathoners. J Sports Sci. 2012;30(12): 1249-1257.

44. McLean SP, Hinrichs RN. Sex differences in the centre of buoyancy location of competitive swimmers. J Sports Sci. 1998;16(4):373-383.

45. Eichenberger E, Knechtle B, Rüst CA, et al. No gender difference in peak performance in ultra-endurance swimming performance analysis of the 'Zurich 12-h Swim' from 1996 to 2010. Chin J Physiol. 2012;55(5):346-351.

46. Eichenberger E, Knechtle B, Knechtle P, Rüst CA, Rosemann T, Lepers R. Best performances by men and women open-water swimmers during the 'English Channel Swim' from 1900 to 2010. J Sports Sci. 2012;30(12):1295-1301

47. Knechtle B, Kohler G. Running performance, not anthropometric factors, is associated with race success in a Triple Iron Triathlon. $\mathrm{Br} J$ Sports Med. 2009;43(6):437-441.

48. Ironman events [webpage on the Internet]. Available from: http://www. ironman.com/events/triathlon-races.aspx\#axzz3373z7rzw. Accessed February 12, 2014.

49. Ironman. Prize money [webpage on the Internet]. Available from: http:// www.ironman.com/triathlon/events/emea/ironman/frankfurt/athletes/ prizemoney. Accessed February 12, 2014.

50. Ironman. The Ironman story [webpage on the Internet]. Available from: http://www.ironman.com/triathlon/history. Accessed February 12, 2014.

51. Stiefel M, Rüst CA, Rosemann T, Knechtle B. A comparison of participation and performance in age-group finishers competing in and qualifying for Ironman Hawaii. Int J Gen Med. 2013;6:67-77.

52. Ironman. Ironman championships [webpage on the Internet]. Available from: http://www.ironman.com/triathlon/events/championships.

53. Deaner RO. Distance running as an ideal domain for showing a sex difference in competitiveness. Arch Sex Behav. 2013;42(3): 413-428.

54. Lombardo MP. On the evolution of sport. Evol Psychol. 2012; 10(1):1-28.

55. Krouse RZ, Ransdell LB, Lucas SM, Pritchard ME. Motivation, goal orientation, coaching, and training habits of women ultrarunners. J Strength Cond Res. 2011;25(10):2835-2842.

56. Frederick C, Ryan RM. Differences in motivation for sport and exercise and their relations with participation and mental health. $J$ Sport Behav. 1993;16(3):124-146.

57. Gill K, Overdorf V. Incentives for exercise in younger and older women. J Sport Behav. 1994;17(2):87-97.

58. Bond K, Batey J. Running for their lives: a qualitative analysis of the exercise experience of female recreational runners. Women Sport Phys Activ J. 2005;14(2):69-82.

59. Hodge K, Allen J, Smellie L. Motivation in masters sports: achievement and social goals. Psychol Sport Exerc. 2008;9(2):157-176.

60. Segar ML, Eccles JS, Peck SC, Richardson CR. Midlife Women's Physical Activity Goals: Sociocultural Influences and Effects on Behavioral Regulation. Sex Roles. 2007;57(11):12837-12849.

61. Masters K, Ogles B. An investigation of the different motivations of marathon runners with varying degrees of experience. J Sport Behav. 1995; 18(1):69-79.

62. Ogles B, Masters K. A typology of marathon runners based on cluster analysis of motivation. J Sport Behav. 2003;26(1):69-85.

63. Knechtle B, Wirth A, Baumann B, Knechtle P, Rosemann T. Personal best time, percent body fat, and training are differently associated with race time for male and female ironman triathletes. Res $Q$ Exerc Sport. 2010;81(1):62-68.

64. Rüst CA, Knechtle B, Knechtle P, Rosemann T. A comparison of anthropometric and training characteristics between recreational female marathoners and recreational female Ironman triathletes. Chin J Physiol. 2013;56(1):1-10. 
65. Knechtle B, Rüst CA, Knechtle P, Rosemann T, Lepers R. Agerelated changes in ultra-triathlon performances. Extrem Physiol Med. 2012;1(1):5.

66. Stiefel M, Knechtle B, Rüst CA, Rosemann T, Lepers R. The age of peak performance in Ironman triathlon: a cross-sectional and longitudinal data analysis. Extrem Physiol Med. 2013;2(1):27.

67. Jeukendrup AE. Nutrition for endurance sports: marathon, triathlon, and road cycling. $J$ Sports Sci. 2011;29 Suppl 1:S91-S99.

68. Herbst L, Knechtle B, Lopez CL, et al. Pacing Strategy and Change in Body Composition during a deca iron triathlon. Chin J Physiol. 2011;54(4):255-263.
69. Sigg K, Knechtle B, Rüst CA, Knechtle P, Lepers R, Rosemann T. Central European triathletes dominate double iron ultratriathlon analysis of participation and performance 1985-2011. Open Access $J$ Sports Med. 2012;3:159-168.

70. Rüst CA, Knechtle B, Knechtle P, Lepers R, Rosemann T, Onywera V. European athletes dominate performances in double iron ultra-triathlons - a retrospective data analysis from 1985 to 2010. Eur J Sport Sci. 2014;14(Suppl 1):S39-S50.

71. Wegelin JA, Hoffman MD. Variables associated with odds of finishing and finish time in a 161-km ultramarathon. Eur J Appl Physiol. 2011;111(1):145-153.

\section{Publish your work in this journal}

Open Access Journal of Sports Medicine is an international, peer-reviewed, open access journal publishing original research, reports, reviews and commentaries on all areas of sports medicine. The manuscript management system is completely online and includes a very quick and fair peer-review system.
Visit http://www.dovepress.com/testimonials.php to read real quotes from published authors. 